\section{O panorama da atenção pré-natal no Brasil e - Programa de Humanização do Pré-natal e Nascimento}

\section{The scenario of pre-natal care in Brazil and the Humanizing of Prenatal Care and Childbirth Program}

Suzanne Jacob Serruya 1

Tânia Di Giácomo Lago 2 José Guilherme Cecatti 3

1 Ministério da Saúde. Departamento de Ciência e Tecnologia. Esplanada dos Ministérios. Bloco G. Ed. Sede, $8^{\circ}$ andar, Sala 834 Brasília, DF, Brasil. CEP: 70.058-900.

2 Faculdade de Ciências Médicas. Santa Casa de São Paulo São Paulo, SP, Brasil.

3 Departamento de Tocoginecologia.

Universidade Estadual de Campinas. Campinas, SP, Brasil.

\begin{abstract}
The purpose of this article was to contextualize historically the advent of the Brazilian Ministry of Health's Prenatal and Birth Humanization Program. Its conceptual, philosophical and operational aspects were pointed out through situation and documental analysis. The Program, launched in June 2000, has as its main strategy to guarantee better access, coverage and quality in prenatal care, birth and puerperal care to pregnant women and newborns from a citizen's rights perspective. The Program is based on the right to humanization of obstetric and neonatal care as the first prerequisite for adequate follow-up, besides establishing criteria for classification of care and promotion of a bond between outpatient care and the moment of delivery, integrated and with interventions that had strong evidence of being effective. The article presents the Brazilian scenario of prenatal care at the end of nineties, discuss the principles of humanization as requirement for the quality of care, reconstitutes the design and launch of the Program, besides commenting practical aspects of its initial evaluation and challenges for the future.
\end{abstract}

Key words Prenatal care, Humanizing delivery, Maternal welfare, Maternal and child health
Resumo O objetivo deste artigo foi contextualizar historicamente a implantação do Programa de Humanização do Pré-Natal e Nascimento do Ministério da Saúde no Brasil, resgatando, através de análise documental e situacional, seus aspectos conceituais, filosófico e operacional. O Programa, lançado em junho de 2000, tem como principal estratégia assegurar a melhoria do acesso, da cobertura e da qualidade do acompanhamento pré-natal, da assistência ao parto e puerpério das gestantes e ao recém-nascido, na perspectiva dos direitos de cidadania. O Programa fundamenta-se no direito à humanização da assistência obstétrica e neonatal como condição primeira para adequado acompanhamento, além de estabelecer critérios para qualificar a assistência e promover o vínculo entre a assistência ambulatorial e o momento do parto, integrados e com intervenções que tivessem fortes evidencias de que são efetivas. O artigo apresenta o panorama da assistência prénatal no Brasil no fim da década de 90, discute os princípios da humanização como requisito para a qualidade da atenção, reconstitui o delineamento e lançamento do Programa, bem como comenta aspectos práticos de sua avaliação inicial e desafios para o futuro.

Palavras-chave Cuidado pré-natal, Humanização 


\section{Introdução}

No final dos anos 90, após quase duas décadas da instituição do Programa de Assistência Integral à Saúde da Mulher (PAISM), a assistência à saúde da mulher no Brasil permanecia com muitas questões a serem enfrentadas. O Ministério da Saúde havia definido a saúde da mulher como prioritária e sistematizou, a partir de três linhas principais de ações, projetos específicos: melhorar a saúde reprodutiva, reduzir a mortalidade por causas evitáveis e combater a violência contra a mulher. 1,2

Para a melhoria da assistência obstétrica, a Área Técnica de Saúde da Mulher, em conjunto com a Secretaria de Assistência à Saúde e Secretaria Executiva, elaborou um plano em três etapas. Na primeira, aumentou a remuneração ao parto normal, instituiu um limite percentual máximo para pagamento de cesáreas por hospital (portaria MS/GM 2.816, de 29 de maio de 1998) e incluiu a remuneração aos procedimentos referentes à analgesia de parto e ao parto realizado por enfermeira obstétrica. Na segunda, foi instituído o Programa de Apoio à Implantação de Sistema Estadual de Referência Hospitalar para a gestação de alto risco (Portaria MS/GM 2.817, de 28 de maio de 1998), com a finalidade de organizar e melhorar a assistência às mulheres com maior risco obstétrico. A terceira etapa tratava especificamente da atenção no pré-natal e ao parto, enfocando a qualidade da assistência, o acesso, também considerado como um indicador da qualidade da atenção, e a humanização da atenção. 1-3

Essas ações tinham como ponto de partida a necessidade de diminuir a morbi-mortalidade materna e melhorar os resultados perinatais, com a perspectiva da humanização como grande fio condutor. Tomando o enfoque de gênero e as questões epidemiológicas como ponto de referência, parecia indispensável retomar compromissos da assistência e promover ações para enfrentar os obstáculos já conhecidos, mas ainda não vencidos. Assim, o objetivo do presente artigo é fazer uma breve análise da assistência pré-natal no Brasil no final da década de 90, cujo panorama justificou o lançamento do Programa de Humanização no Pré-Natal e Nascimento (PHPN).

\section{Panorama da assistência pré-natal no Brasil}

No Brasil, o acesso ao pré-natal como condição primeira para a assistência apresenta ainda importantes diferenciais por região, residência e escolari- dade. Na Pesquisa Nacional de Demografia e Saúde (PNDS) em 1996, da Sociedade Civil Bem-Estar Familiar no Brasil (BEMFAM) 4 14,3\% das mulheres que tiveram filhos nos cinco anos que antecederam a pesquisa, não haviam recebido nenhuma consulta de pré-natal, sendo $7,6 \%$ na cidade e $30,3 \%$ no campo. O Rio de Janeiro apresentava a menor taxa de mulheres sem cobertura pré-natal, de $3,8 \%$, em contraste com a falta de consultas para $25,2 \%$ das mulheres nordestinas e $17,1 \%$ das mulheres na Região Norte. Em relação à escolaridade, $42,6 \%$ das mulheres sem prénatal eram analfabetas e $27 \%$ haviam cursado apenas de um a três anos de estudo, totalizando $69,6 \%$. Ou seja, as mulheres que tinham problemas de acesso ao pré-natal estavam nos locais e regiões mais pobres e tinham menos possibilidade de educação formal, convergindo diferentes graus de exclusão social. Por outro lado, $77 \%$ das mulheres realizaram mais de quatro consultas e, destas, $47,5 \%$ realizaram mais de sete consultas. A mediana do número de consultas em todos os lugares pesquisados esteve sempre acima de seis, mas mantiveram-se também as diferenças encontradas para a cobertura: as menores medianas foram as da zona rural e das Regiões Norte e Nordeste. 4

Os dados coletados na PNDS em 1996 são semelhantes aos declarados no Sistema de Nascidos Vivos (SINASC) nos últimos anos. Em 1997, 49,8\% e em $1998,49,5 \%$ das mulheres declararam ter realizado mais de sete consultas de pré-natal. As desigualdades regionais permanecem: no Sudeste o percentual encontrado é de $58,6 \%$, e de $38,6 \%$ na região Norte. 5

Analisando as informações do Sistema Único de Saúde (SUS), encontra-se um incremento considerável do número de consultas por mulher que realiza o parto no SUS, nos últimos anos. 6 O aumento mais importante ocorreu entre 1997 e 1998, pela inclusão do acompanhamento pré-natal no conjunto de ações básicas que devem ser desenvolvidas pelos municípios. Tomando como base a razão entre o número de consultas no país e o número de mulheres que realizam o parto no SUS, permanecem as Regiões Norte e Nordeste com os menores valores (Figura 1). Apesar do número absoluto de consultas ter aumentado, a razão nacional em 2000 era de quatro e, para que todas as mulheres conseguissem realizar seis consultas, seria necessário um incremento de mais de cinco milhões de consultas de pré-natal por ano. Este crescimento, de $50 \%$ para o país, deveria representar um aumento de $105 \%$ para a Região Norte e de $150 \%$ para os estados do Nordeste (Tabela 1). 
Razão entre o número de consultas de pré-natal e parto. Sistema Único de Saúde (SUS) no Brasil e Regiões de 1995 a 2000.

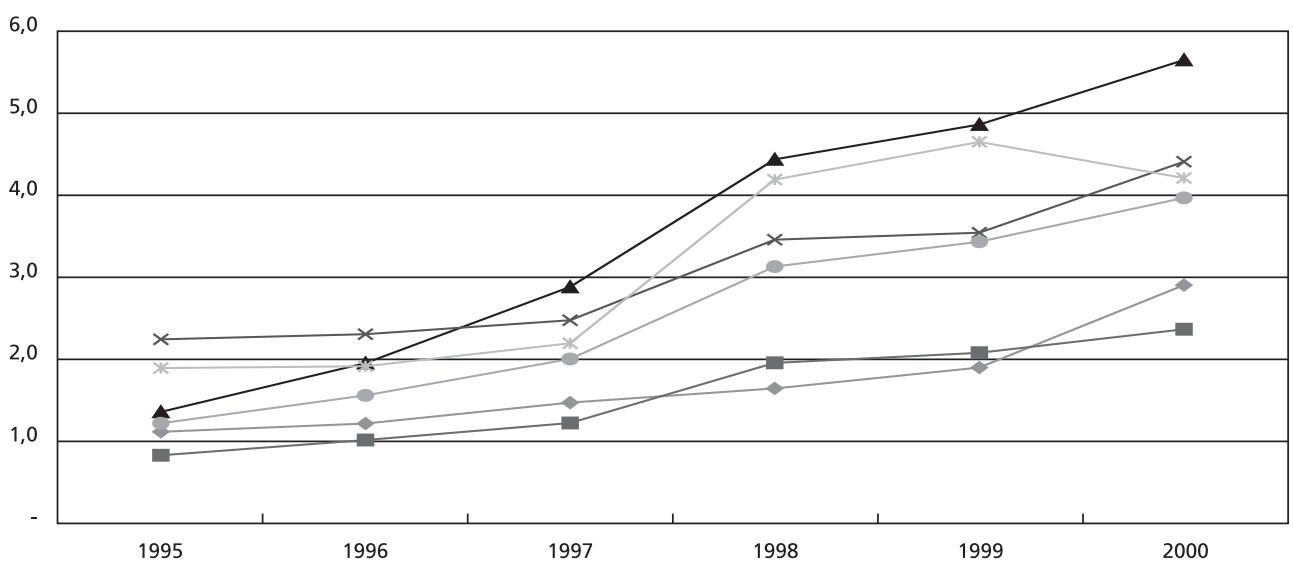

Número de partos, consultas de pré-natal necessárias, realizadas, déficit e crescimento percentual necessário para Brasil e Regiões. Sistema Único de Saúde (SUS), 2000.

\begin{tabular}{|c|c|c|c|c|c|}
\hline Região & $\begin{array}{l}\text { Total de } \\
\text { partos }\end{array}$ & $\begin{array}{c}\text { Consultas } \\
\text { necessárias }\end{array}$ & $\begin{array}{l}\text { Consultas } \\
\text { realizadas }\end{array}$ & Déficit & $\begin{array}{c}\text { Crescimento } \\
\text { necessário (\%) }\end{array}$ \\
\hline Norte & 243.214 & 1.459 .284 & 709.330 & 749.954 & 105,0 \\
\hline Nordeste & 878.686 & 5.270 .316 & 2.086 .300 & 3.184 .016 & 152,0 \\
\hline Sudeste & 880.224 & 5.281 .344 & 5.005 .882 & 275.462 & 5,5 \\
\hline Sul & 329.066 & 1.974 .396 & 1.460 .175 & 514.221 & 35,0 \\
\hline Centro-Oeste & 174.460 & 1.046 .760 & 739.098 & 307.662 & 41,0 \\
\hline Brasil & 2.505 .350 & 15.032 .100 & 10.000 .785 & 5.031 .315 & 50,0 \\
\hline
\end{tabular}

Fonte: Ministério da Saúde. Secretaria de Políticas de Saúde. Brasília (DF); 2003.1

Um estudo recente sobre as desigualdades de saúde no Brasil apresenta, entre os indicadores de qualidade selecionados, o percentual de mulheres que receberam pelo menos seis consultas pré-natal.7 Na correlação entre taxa de pobreza e cobertura prénatal, os autores afirmam que esta é inversamente proporcional, embora ocorra interferência de outros fatores. O resultado dessa correlação apontou que a maioria dos estados com cobertura de pelo menos seis consultas de pré-natal apresentava um nível econômico compatível com o esperado para a realização dessas consultas e que, a cada redução de $10 \%$ na taxa de pobreza, haveria o aumento de $7 \%$ na cobertura pré-natal, ratificando o vínculo entre assistência e renda.

Deste modo, o acesso aparece como um aspecto 
fundamental a ser considerado em uma estratégia para qualificar a assistência, ${ }^{8}$ uma vez que os dados em diferentes estudos apontam-no como um lócus de vulnerabilidade da assistência, onde a convergência de outras variáveis como renda, região, localização e anos de estudo potencializam a exclusão da mulher.

$\mathrm{Na}$ análise da qualidade da assistência prestada propriamente dita, na ausência de informações que detalhem o conteúdo das consultas e sua adequação, consideram-se quatro aspectos representativos da assistência que vão além do pré-natal e incluem também fatores de saúde perinatal, tomados como indicadores: a taxa de captação precoce, a incidência de sífilis congênita, a distribuição das causas de morte materna no país e o número de consultas de puerpério.

A recomendação de se iniciar o pré-natal assim que a gestação seja diagnosticada objetiva fortalecer a adesão da mulher ao pré-natal e diagnosticar eventuais fatores de risco. Os dados da PNDS 4 apontam que $66 \%$ das mulheres iniciaram o pré-natal até o terceiro mês. As diferenças em relação às regiões e localização são as mesmas que as encontradas para a questão do acesso: assim, a taxa de captação precoce rural é $45,7 \%$ e a urbana de $72,7 \%$ e as Regiões Nordeste e Norte apresentam as menores taxas, de $51,9 \%$ e $55,7 \%$ respectivamente.

Em 1999, 4086 casos de sífilis congênita foram notificados no país. Como a região Sul notificou quase $50 \%$ desses casos (2184) e a Região Norte apenas 91 casos, a avaliação deve levar em conta a provável grande sub-notificação que ocorre, uma vez que a qualidade do dado está sujeita à capacidade do sistema de vigilância epidemiológica, em cada região, para a realização de todas etapas: detectar, notificar, investigar e confirmar os casos. Esses dados, para os anos recentes e por região, estão na Tabela 2. A sífilis é uma condição patológica cujo diagnóstico e tratamento podem ser realizados com baixo custo e nenhuma dificuldade operacional, uma vez que a imensa maioria dos laboratórios dispõe de tecnologia para a realização do exame. Os dados mostram que, surpreendentemente, o número de casos de sífilis congênita é igual para o grupo que realizou pré-natal e para o que não realizou. 6

Tabela 2

Número de casos confirmados de sífilis congênita* para Brasil e regiões, 1998 e 1999.

\begin{tabular}{lrrrr}
\hline & & 1998 & & 1999 \\
\cline { 2 - 5 } Região & \multicolumn{1}{c}{$\mathrm{n}$} & $\%$ & $\mathrm{n}$ & \\
\hline Norte & 43 & 1,2 & 91 & 2,2 \\
Nordeste & 1.208 & 32,0 & 1.034 & 212 \\
Sudeste & 303 & 8,0 & 2.184 & 5,2 \\
Sul & 1.856 & 49,1 & 565 & 13,8 \\
Centro-Oeste & 368 & 100,0 & 4.086 & 100,0 \\
Brasil & 3.778 & & & \\
\hline
\end{tabular}

* Casos diagnosticados nos dois primeiros anos de vida.

Fonte: Ministério da Saúde Centro Nacional de Epidemiologia. Brasília, DF; 2003.6

No Brasil, a hipertensão na gravidez corresponde à principal causa de morte materna obstétrica direta nos últimos anos, seguida pelas demais causas diretas (Tabela 3). O diagnóstico da hipertensão, principalmente da crônica, também é importante na prevenção de quadros como os de descolamento prematuro da placenta, causa importante de óbitos maternos e fetais. Como doença oligo ou assintomática, sua detecção torna indispensável o uso de uma técnica muito simples e de baixíssimo custo, a aferição da pressão arterial em todas as consultas de pré-natal. O diagnóstico com a realização desse procedimento e condutas corretas permitiria salvar muitas mulheres. É por essa razão que, em países desenvolvidos, as baixas taxas de mortalidade materna contabilizam predominantemente causas indiretas, demonstrando a eficácia de se prevenir essas mortes. 3 
Principais causas de mortalidade materna. Brasil, 1995 a 1999.

\begin{tabular}{|c|c|c|c|c|c|c|c|c|c|c|}
\hline \multirow{2}{*}{ Causas } & \multicolumn{2}{|c|}{1995} & \multicolumn{2}{|c|}{1996} & \multicolumn{2}{|c|}{1997} & \multicolumn{2}{|c|}{1998} & \multicolumn{2}{|c|}{1999} \\
\hline & $\begin{array}{l}\text { Óbitos } \\
\text { (n) }\end{array}$ & Razão & $\begin{array}{l}\text { Óbitos } \\
\text { (n) }\end{array}$ & Razão & $\begin{array}{l}\text { Óbitos } \\
(n)\end{array}$ & Razão & $\begin{array}{l}\text { Óbitos } \\
\text { (n) }\end{array}$ & Razão & $\begin{array}{l}\text { Óbitos } \\
\text { (n) }\end{array}$ & Razão \\
\hline Hipertensão & 442 & 12,8 & 332 & 9,6 & 383 & 11,0 & 426 & 12,1 & 459 & 13,1 \\
\hline Hemorragia & 310 & 9,0 & 270 & 7,8 & 248 & 7,1 & 236 & 6,7 & 319 & 9,1 \\
\hline Infecção Puerperal & 101 & 2,9 & 126 & 3,6 & 139 & 4,0 & 138 & 3,9 & 113 & 3,2 \\
\hline Aborto & 127 & 3,7 & 97 & 2,8 & 118 & 3,4 & 77 & 2,2 & 105 & 3,0 \\
\hline $\begin{array}{l}\text { Doença do Aparelh } \\
\text { Complicações GPP }\end{array}$ & 79 & 2,3 & 90 & 2,6 & 171 & 4,9 & 201 & 5,7 & 136 & 3,9 \\
\hline Outras & 576 & 16,7 & 605 & 17,5 & 792 & 22,8 & 964 & 27,6 & 657 & 18,7 \\
\hline Total & 1.635 & 47,7 & 1.520 & 44,1 & 1.851 & 53,4 & 2.039 & 58,5 & 1.789 & 51,0 \\
\hline
\end{tabular}

Razão por 100.000 NV

A assistência à mulher na gestação só deveria ser considerada como concluída após a consulta puerperal. O seguimento clínico após a gestação é imperativo por diferentes razões, como o estabelecimento de condutas para garantir o adequado intervalo interpartal, que protege a mulher e melhora os resultados perinatais, com a orientação para a introdução de método contraceptivo, assim como avaliação que permita detectar importantes alterações como a anemia e os estados depressivos. Os registros no Sistema de Informações Ambulatoriais (SAI) do SUS apresentam um número muito baixo de consultas puerperais, de 1250 em 2000.6 Ainda que seja provável que tal número esteja subestimado e que mais mulheres retornam para essa avaliação sem que o registro desse procedimento ocorra, de qualquer forma a atenção ao puerpério prestada no Brasil é ínfima, sem contar nos aspectos qualitativos, freqüentemente subestimados. Entretanto, a cobertura vacinal realizada no primeiro mês de vida (BCG) é de $100 \%$ há pelo menos seis anos em todo país, confirmando que a mulher volta ao serviço de saúde apenas para o cuidado com o recém-nascido, espelhando a falta de articulação das ações básicas e o viés de gênero. 5

\section{A humanização como princípio da qualidade de atenção}

Além dessas questões, mais objetivas e quantificáveis, mostrando um quadro crítico da atenção ao pré-natal no país até o ano de 2000 , é provável que esta impressão de má qualidade esteja também relacionada à falta de práticas humanizadas, embora praticamente inexistam estudos avaliando globalmente a qualidade técnica do pré-natal no território nacional. Um exemplo poderia ser chamado "alta" do pré-natal. A falta de atendimento ambulatorial no final da gestação, no momento de maior probabilidade de intercorrências obstétricas, é fator importante na determinação dos resultados maternos e perinatais. Era comum que o serviço ambulatorial de pré-natal "desse alta" à mulher e apenas a orientasse a procurar o hospital no momento do parto ou se aparecesse alguma complicação, deixando sob sua responsabilidade conseguir a internação. A falta de acolhimento nas unidades e a "alta do pré-natal" podem ser interpretadas como emblemáticas da desumanização dos serviços e, em conjunto com os demais indicadores que apontavam para um pré-natal ainda insuficiente e com sérias restrições de qualidade, mostravam, de maneira inequívoca, a necessidade de mudança. Ratifica este quadro, o fato de que, apesar dos dados disponíveis apontarem um aumento considerável nos últimos anos no número de consultas por mulher, 1,4 esse incremento não resultou em impacto considerável nos óbitos maternos declarados, mesmo nas regiões Sudeste e Centro-Oeste que têm média maior que quatro consultas há quatro anos. 1,2

A questão da falta de vínculo entre a assistência pré-natal e a do parto leva as mulheres, em trabalho de parto, a uma peregrinação à procura de vagas nos hospitais. Além disso, a maioria das mortes maternas ocorre perto do parto, demandando intervenções que 
garantam melhor assistência nesse período. Nesse panorama da situação obstétrica, a crença de que existe uma desumanização em um momento tão importante e, principalmente, o direito que toda mulher tem de garantia ao atendimento foram consideradas como questões emblemáticas a serem enfrentadas.

Embora as causas para a manutenção desse quadro sejam diversas e provavelmente não consensuais, é provável que se destaquem a postura mercantilista dos serviços e a falta de compromisso profissional. Dos hospitais que atendem ao parto no Brasil, cerca de 4700 , ou $63 \%$, são conveniados ao SUS, ou seja, apenas "vendem" serviços e raramente têm preocupações, de fato, com os resultados assistenciais. Por outro lado, os profissionais de saúde carecem de capacitação técnica permanente, uma vez que a grande maioria, após a graduação, não volta a se atualizar, além de inúmeras questões que giram em torno do processo de trabalho médico e que fogem do escopo desta abordagem. Por um lado é verdade que tal panorama pudesse ser assumido como resultante de uma opinião pessoal, dada à existência de estudos que avaliem sistematicamente todos esses elementos de forma prospectiva, também é verdade que essa impressão é relatada pela grande maior parte de gestores e técnicos que avaliam a saúde materno-infantil no país.

Ampliando esta análise do panorama obstétrico chegava-se à avaliação do modelo assistencial vigente no país, que refletia um determinado paradigma conceitual de atenção. Em torno do consenso de que a assistência obstétrica precisava melhorar em todos os aspectos (acesso, acolhimento, qualidade, resolutividade), a questão era a discussão de uma atenção baseada em princípios tecnocráticos versus o paradigma da assistência humanizada. ${ }^{9}$ Tais questionamentos, relativos ao modelo praticado na assistência, refletiam um debate sobre as práticas clínicas em geral, na qual se partia da percepção de que as "técnicas" empregadas obedeciam a uma lógica de valores sociais em que a "tecnologia" aparece como o ícone máximo na assistência. Com esse pressuposto, a organização dos serviços e a interação entre profissionais de saúde e pacientes desenha-se com princípios mercadológicos e de produtos.

Tais práticas clínicas estavam fortemente embasadas em questões culturais deste modelo normatizador e pouco fundamentadas do ponto de vista científico. O modelo tecnocrático obedecia a uma padronização no cuidado e, como os modelos fabris, os "produtos" deveriam seguir uma linha de produção e, portanto, não "atrapalhar" a ordem médica hospitalar. Embora as resistências às imposições médicas tenham sempre estado presentes, para as mulheres elas se concretizaram no momento do parto, uma vez que é nesse cenário que ocorrem as maiores intervenções, no sentido de padronizar, ordenar e obter "bons produtos". 9,10

O reconhecimento de que as práticas médicas caminharam na direção da "expropriação da saúde" é bem sinalizado em Illich (1975: 33) 11 que, partindo do conceito de doença iatrogênica como a que "... engloba todas as condições clínicas das quais os medicamentos, os médicos e os hospitais são os agentes patogênicos." se analisa nos acontecimentos deflagrados historicamente a construção de uma prática médica expropriadora. Ele discute, nesta abordagem, seus diferentes aspectos, clínico, social e estrutural, apontando de que maneira a saúde torna-se um produto de consumo e a medicalização uma constante norma da vida e da morte.

Essa discussão também é realizada por Foucault12 que, na análise do nascimento da medicina social, refaz conceitualmente seu percurso histórico e aponta que o controle social sobre os indivíduos começou no corpo, entendido como uma realidade bio-política e a medicina como uma estratégia biopolítica. Assim, a possibilidade de interferir concretamente na vida pública e privada da sociedade foi estabelecida em espaços, tempos e contextos sociais diferentes, cujo ponto de contacto materializa-se através de um poder que pressupõe um saber. ${ }^{13}$ Tais reflexões permitem compreender que os modelos atuais de assistência estão fortemente marcados por esse paradigma e a retomada de questões, como a autonomia e humanização, pressupõe a discussão de referenciais filosóficos. Não será possível mudar a assistência sem ampliar esse olhar e discutir esses conceitos. ${ }^{14}$ Sem um entendimento amplo, por exemplo, da questão da relação entre o profissional de saúde e a mulher, a assimetria desta relação é explicada por um olhar reducionista e maniqueísta: os profissionais de saúde não são comprometidos, as pacientes são "coitadinhas".

A introdução dessa discussão na assistência obstétrica é historicamente recente no país. Reflete, em primeiro lugar, o questionamento e a negação dessas práticas relacionadas ao parto e à assistência à mulher, iniciados nos países desenvolvidos por grupos de mulheres e profissionais de saúde. O descontentamento com este modelo iniciava-se pelo excesso de intervenção no parto normal e crescentes taxas de cesárea. ${ }^{9,15}$ Sedimentava-se uma postura do movimento de mulheres, exemplificada, desde a década de 70, na palavra de ordem "nosso corpo nos pertence" que pressupunha a assistência ao parto, sem dúvida a mais exemplar, recolocando questões como: a quem o parto pertence e em que são 
baseadas as práticas realizadas nesse momento, tão significantes do ponto de vista sócio-cultural.16,17

Historicamente, essa discussão ganhou grande impulso quando, em 1985, em Fortaleza, representantes e convidados da Organização Mundial da Saúde e da Organização Pan-Americana de Saúde reuniram-se e fizeram recomendações para um conjunto de práticas consideradas de "rotina", questionando seu papel na assistência ao parto. O reconhecimento de que o nascimento, na grande maioria dos casos, necessita apenas de observação deveria ser geral e o uso de técnicas resultantes do progresso da Obstetrícia deveria ser criterioso e não indiscriminado, com base no princípio de que nenhuma técnica é intrinsecamente boa ou ruim, e sua aplicação não deve ser automaticamente rotina sem as devidas indicações, mesmo para as práticas consideradas mais simples. Uma questão central que aparece é que o excesso de intervenções é responsável por um conjunto de desvantagens que incluem desde o desperdício de recursos até importantes iatrogenias. $15,18,19$

Neste momento começa a se concretizar uma postura ainda fragmentada, mas já com grande representatividade, de absoluta negação ao modelo tecnocrático. De maneira antagônica ao "tecnicismo", várias vozes levantaram-se reclamando a retomada de partos "naturais", com opiniões diferentes acerca da cena do parto, atores e cenários. ${ }^{9}$ Surge um forte e imediato consenso: é necessário "humanizar" o parto e o nascimento. Desse modo, a proposição da humanização é, acima de tudo, o reconhecimento da autonomia da mulher, enquanto ser humano, e da óbvia necessidade de tratar esse momento com práticas que, de fato, tenham evidências e permitam aumentar a segurança e o bem-estar da mulher e do recém-nascido, respeitando as suas escolhas. ${ }^{20}$

Essa corrente a favor de uma nova prática obstétrica obteve importante reforço, político e técnico, quando, em 1996, a Organização Mundial da Saúde publicou um guia para a assistência ao parto normal, apresentando as recomendações, baseadas em evidências, sobre as práticas relacionadas ao parto normal, 15 classificadas em categorias, de acordo com o nível de evidência científica disponível segundo a classificação de Mulrow. ${ }^{21}$ As recomendações objetivam garantir os direitos das mulheres e diminuir as intervenções desnecessárias, questionando procedimentos realizados sem nenhum critério científico, apenas por hábito ou rotina. 15 Esse guia, posteriormente traduzido ao português, foi bem recebido em todo mundo e, especialmente, na América Latina e no Brasil. Representou ainda a inspiração para a elaboração do respectivo manual técnico pelo Ministério da Saúde. 19
$\mathrm{Na}$ literatura não existe um consenso em torno do conceito de "humanização". Em 2001, na Conferência Internacional da Humanização do Nascimento, realizada em Fortaleza, houve o anúncio das definições de "humanização" e "cuidado humanizado", acordadas entre as diversas lideranças presentes que, amplas, objetivam contextualizar sob diferentes aspectos esta questão.22 Atualmente, a discussão sobre humanização e seus principais aspectos, como a autonomia e direitos, já faz parte de uma série de instâncias. Entretanto, para as mulheres, trata-se de uma situação que tem marcado o conjunto das vivências femininas, em que a assistência ao parto é simbolicamente o foco de maior medicalização e resistência. Também na assistência pré-natal ocorre uma expropriação da autonomia feminina e estão presentes todos os pilares do modelo tecnocrático: as mulheres são vistas como objeto, fora de contexto, onde a "máquina" é examinada e não se estabelece nenhum vínculo com o profisssional. As ações praticadas são de responsabilidade dos profissionais que supervalorizam as mais técnicas e mantêm, com intervenções determinadas, a hierarquia e padronização das rotinas.

\section{O marco conceitual do programa}

O contraponto a esse modelo pressupõe que a mulher é um ser único, complexo, com sentimentos e autonomia. Os profissionais de saúde devem informar e dividir as decisões e responsabilidades com elas, equilibrando a tecnologia e o humanismo, abertos a outras práticas e enfocando a prevenção. ${ }^{9} \mathrm{Re}$ sumidamente, foram essas as razões e as características do sistema que levaram à necessidade de uma estratégia que sensibilizasse gestores e profissionais para tentar melhorar a assistência obstétrica no país com uma visão ampla e crítica. Assim, no conjunto de medidas tomadas pelo Ministério da Saúde, em um plano com distintas etapas, o lançamento do Programa de Humanização no Pré-Natal e Nascimento deveria somar-se a outras importantes estratégias já em andamento para a melhoria da assistência obstétrica. 23

A proposta dessa estratégia tinha como primeiro objetivo rediscutir a atenção pré-natal na assistência básica. Para isso, era indispensável remeter às questões aqui expostas e, num contexto de direitos e resultados, propor novo modelo. Do ponto de vista conceitual, essa estratégia deveria contemplar, a partir de um olhar de gênero, as questões da humanização e as chamadas "técnicas", entendendo que a assistência, complexa, não separa estas instâncias. Isso 
implicava em reconhecer que, para que a assistência pudesse efetivamente melhorar, não deveriam ser operadas ações separadas e que a idéia do todo não poderia ser perdida.

A partir deste marco conceitual, a portaria GM n. ${ }^{\circ} 569$, que instituiu o Programa de Humanização do Pré-Natal e Nascimento traz, em seu primeiro "considerando", o seguinte texto:

"...Considerando que o acesso das gestantes e recém-nascidos a atendimento digno e de qualidade no decorrer da gestação, parto, puerpério e período neonatal são direitos inalienáveis de cidadania ..." (Ministério da Saúde; 2000: 5).23

Esse pressuposto registra a questão dos direitos das mulheres e dos deveres constituídos pelo Estado em um instrumento normativo cujo teor, em geral restringe-se a instruções programáticas ou de regulação do sistema. O estabelecimento desse referencial em um documento oficial teve como objetivo demarcar o princípio do direito como marco conceitual do programa. $\mathrm{O}$ descaso com a gestação e suas intercorrências se dá em um contexto de "aceitação" do óbito materno como destino inevitável e a percepção da reprodução apenas como evento biológico "feminino", sem qualquer conotação social e política. Desse modo, o reconhecimento do dever e do direito busca estabelecer um campo político para essa questão, antes de anunciar as medidas "técnicas".

A seguir, os dois outros considerandos da mesma portaria referem-se à redução das taxas de morbimortalidade materna e perinatal, às questões de acesso, cobertura e qualidade, reunindo os três principais temas do Programa. Os demais pontuam as questões da assistência à gestação, a partir das medidas já implantadas, destacando que o objetivo é "garantir a integralidade assistencial", por meio do aprimoramento em todos os níveis de atenção. No corpo da portaria, o artigo $2^{\circ}$, que traz os princípios e diretrizes, explicita a questão do direito enunciada no primeiro considerando:

"... Art. $2^{\circ}$ Estabelecer os seguintes princípios e diretrizes para a estruturação do Programa de Humanização no Pré-natal e Nascimento: a - toda gestante tem direito ao acesso a atendimento digno e de qualidade no decorrer da gestação, parto e puerpério; $b$ - toda gestante tem direito de saber e ter assegurado o acesso à maternidade em que será atendida no momento do parto; $c$ - toda gestante tem direito à assistência ao parto e ao puerpério e que esta seja realizada de forma humanizada e segura." (Ministério da Saúde; 2000: 7).23

Na finalização dos princípios, ratifica que: "... as autoridades sanitárias dos âmbitos federal, estadual e municipal são responsáveis pela garantia dos direitos enunciados nas alíneas acima..." (Ministério da Saúde; 2000: 8).23

A presença de formulações não usuais em portarias ministeriais foi a tônica do Programa em um primeiro momento, mobilizando as discussões para o resgate de uma assistência mais comprometida com os princípios aqui anunciados.

\section{O Programa de Humanização do Pré-Natal e Nascimento}

Como o Programa está compreendido em um projeto de aprimoramento da assistência obstétrica integralmente, no seu desenho institucional estão contempladas outras instâncias da gestão indispensáveis para a consecução dos objetivos anunciados. Desse modo, a Portaria GM No 569 apresenta a estrutura do programa com três componentes:

"... Componente I - Incentivo à Assistência Prénatal, que objetiva a assistência pré-natal adequada, com critérios mínimos recomendados pelo Programa, instituindo, para tanto, incentivos financeiros a serem transferidos aos municípios que aderirem ao Programa.

Componente II - Organização, Regulação e Investimentos na Assistência Obstétrica e Neonatal, que objetiva o desenvolvimento de condições técnicas e operacionais para a organização e regulação da assistência obstétrica e neonatal, através da estruturação de Centrais de Regulação e de sistemas móveis de atendimento pré e inter-hospitalares e financiamento a hospitais públicos e filantrópicos integrantes do Sistema Único de Saúde.

Componente III - Nova Sistemática de Pagamento da Assistência ao Parto - buscando a melhoria do custeio da assistência ao parto realizado nos hospitais integrantes do Sistema de Informações Hospitalares - SIH/SUS, elevando o valor e a forma de remuneração dos procedimentos da tabela relativos ao parto ..." (Ministério da Saúde, 2000: 18).23

A análise dos componentes II e III, assim como das outras medidas para a assistência obstétrica, foge do escopo da presente avaliação. Considerou-se fundamental avaliar a experiência da implantação do Programa de Humanização no Pré-natal e Nascimento do Ministério da Saúde, no Brasil, cujo estabelecimento, enquanto estratégia para a melhoria de acesso e qualidade da assistência, teve como fio condutor a 
questão da humanização da atenção e o respeito aos direitos reprodutivos. ${ }^{24} \mathrm{O}$ olhar de gênero permitiu desatrelar da situação materna o caráter de destino inevitável e pensar em estratégias que empoderassem as mulheres, revendo, na perspectiva de humanização, o seu papel de protagonista na gestação e no parto. A humanização necessariamente compreende o resgate da autonomia e relações simétricas entre profissionais e mulheres.

O Programa instituiu critérios para qualificar a assistência e um sistema de informações, o SISPRENATAL, para monitorar o cumprimento dos mesmos e acompanhar indicadores de qualidade definidos pelo PHPN.23 Até dezembro de 2002, 72\% dos municípios do país aderiram ao Programa e, em junho de 2002, 3183 desses municípios apresentaram produção, constituindo um banco de dados com 720.871 mulheres. O indicador de cobertura do programa mostrou um incremento importante entre 2001 (9,25\%) e 2002 (27,92\%), com o cadastramento de 555.979 mulheres no segundo ano, a maioria inscrita com até 120 dias de gestação. O percentual de mulheres que realizou seis ou mais consultas de prénatal foi próximo de $20 \%$ e a conjugação deste critério com a consulta puerperal e/ou os exames obrigatórios, reduziu esse percentual para a metade. O registro da vacina antitetânica cresceu $100 \%$ de 2001 para 2002, enquanto o percentual de solicitações de exames para a detecção de sífilis foi a metade em relação a solicitações de exames para o HIV nesses dois anos. Apenas 2\% das mulheres cadastradas em 2001 e 5\% em 2002 realizaram o conjunto de todos os critérios assistenciais estabelecidos. ${ }^{24}$ Apesar dos baixos percentuais registrados, a comparação dos indicadores de qualidade de assistência revelou crescimento de 2001 para 2002.

A discussão da assistência pré-natal também foi fortalecida por um conjunto de importantes conferências internacionais ocorridas nas duas últimas décadas, que estabeleceram metas em vários campos, com prioridade para a saúde. Como a ocorrência de mortes maternas é questão fundamental, a discussão de propostas efetivas para melhorar essa assistência foi recorrente, incorporando na agenda da saúde os direitos da mulher.22,25-29

No cenário de intervenções de saúde pública voltadas para a saúde da mulher, o PHPN apresenta-se como uma estratégia singular. A maioria dos programas para a saúde de gestantes foi elaborada em países desenvolvidos, mesmo quando o seu formato visava atender as necessidades de países em desenvolvimento e, em geral, foram iniciativas internacionais baseadas em metas para a redução da morte materna. Embora praticamente todos os países ofereçam a assistência pré-natal, a avaliação indireta mais comumente realizada é o monitoramento da mortalidade materna. Na literatura disponível, até onde foi possível consultar, não se encontrou nenhum programa no formato do PHPN. $\mathrm{O}$ ineditismo é dado por vários aspectos: foi uma intervenção planejada em um país em desenvolvimento, estruturada com medidas para as diferentes instâncias da assistência, com um desenho que buscava mudar um paradigma na atenção, com estabelecimento de critérios mínimos, com o incentivo financeiro rigorosamente atrelado ao cumprimento desses critérios e com um sistema de informações que permitia o monitoramento e a avaliação da assistência em curso.

Ainda existe um longo caminho no sentido de incluir na rotina a avaliação sistemática das atividades da saúde. A grande maioria dos gestores ainda não incorporou o sistema de informações enquanto instrumento de gestão. Nesse sentido, o Programa deverá necessariamente demandar esforços para que ele seja utilizado como ferramenta de monitoramento das ações e a incorporação da avaliação como atividade regular, possibilitando aos executores, na ponta do sistema, intervir e corrigir rumos.

O PHPN foi planejado e discutido como uma política nacional em atenção aos direitos das mulheres, buscando efetivar uma ação fundamental para a melhoria da qualidade obstétrica e a redução da mortalidade materna e perinatal, em conjunto com outras medidas adotadas para alcançar esse objetivo como o limite percentual no pagamento das cesáreas e a implantação de um sistema de atenção à gestação de alto risco. Durante sua implantação, mobilizou, em tempo relativamente curto, quase quatro mil municípios e permitiu a ratificação de muitas hipóteses sobre a assistência pré-natal, com um macro diagnóstico da saúde materna. 24

Em municípios onde o PHPN foi prioridade, várias iniciativas foram sedimentadas em relação à qualidade da atenção. Nas inúmeras observações realizadas, o pré-natal foi resgatado como estratégia importante e seu monitoramento cuidadosamente feito, inclusive com a implementação de atividades recomendadas, mas não obrigatórias, como a visita da gestante à maternidade e a presença de acompanhantes no momento do parto. As coordenações locais referiam-se à instituição do Programa como a possibilidade de concretizar várias atividades para a qual não se sentiam empoderadas. Seguramente, existem ainda muitos aspectos dessa estratégia que devem ser conhecidos e os seus desdobramentos avaliados. 


\section{O futuro}

A abordagem utilizada neste artigo focalizou os aspectos conceituais da implantação do Programa, constituindo-se no primeiro estudo sobre essa estratégia, com o objetivo de registrar o histórico da iniciativa, juntamente com outra publicação que aborda os resultados preliminares dos dois primeiros anos do Programa. ${ }^{24}$ Certamente, releituras desse processo poderão ser mais enriquecedoras à medida que estejam mais perto dos atores envolvidos e permita maior proximidade da problemática, em contextos bem determinados.

Uma das discussões fundamentais, e ainda incipiente, é sobre os aspectos da humanização propriamente dita que nomeou o Programa e que podem ser diferentemente percebidos no percurso da sua implantação. A concepção que orientou a criação do Programa e a abordagem realizada neste artigo pressupõe que a humanização da assistência pré-natal requer, antes de tudo, o cumprimento desse conjunto de procedimentos básicos a fim de prevenir agravos na gestação e garantir o direito fundamental de toda mulher à experiência da maternidade de maneira segura. Contudo, muitas questões merecem análise e certamente duas ações merecem avaliações imediatas e amplas: o vínculo entre as duas instâncias assistenciais e a presença das práticas humanizadoras na atenção ao parto. As duas problemáticas, pela natureza e complexidade, demandam estudos operacionais com método qualitativo, capazes de incluir olhares dos diversos atores envolvidos: gestores, profissionais de saúde e principalmente mulheres.

Com a correta implementação do Programa, espera-se avançar em resultados maternos e perinatais. Para o conhecimento deste impacto, além dos tradicionais indicadores de mortalidade materna e perinatal, serão de fundamental importância indicadores de morbidade e de satisfação das gestantes, uma vez que fornecerão elementos relevantes para a correção da estratégia. Um estudo recém publicado avaliou a qualidade da assistência pré-natal no Brasil através da opinião das mulheres usuárias do serviço, apontando para uma percepção de qualidade dos serviços, de maneira contrária à impressão compartilhada pelos técnicos e gestores. ${ }^{30} \mathrm{~A}$ análise preliminar, da qual este artigo faz parte, aponta para um conjunto de questões que deverá ser objeto de novas intervenções, parte maior dos gestores locais, com mais instrumentos para corrigirem rumos na execução das políticas, e parte com os gestores estadual e federal, que devem coordenar esforços para resolver questões graves e crônicas como a falta de leitos de terapia intensiva e a efetivação de um sistema de referência e contra-referência.

Nessa direção, é importante ressaltar que o PHPN necessita de ampla articulação interna, nos três níveis, notadamente com o Programa de Saúde da Família, uma vez que a presença de uma equipe desse programa já garantiria a realização do pré-natal. Essa articulação permitiria fortalecer ambas as iniciativas e eliminar etapas burocráticas, além de outros passos realizados em cada município para a implantação de cada um dos programas.

Por fim, é preciso dizer que esta estratégia do Ministério da Saúde, ao enfrentar a problemática da mortalidade materna e perinatal, a partir do pressuposto de direitos humanos e princípios de humanização, deverá, pela sua magnitude e importância, inspirar outros países em desenvolvimento a iniciativas semelhantes que possam garantir, em diferentes locais, mais segurança e bem-estar para mulheres e recém-nascidos.

\section{Referências}

1. Ministério da Saúde. Secretaria de Políticas de Saúde. O desafio de construir e implementar políticas de saúde: relatório de gestão 2000-2002. Brasília (DF): O Ministério; 2002.

2. Leão EM, Marinho LFB. Saúde das mulheres no Brasil: subsídios para as políticas públicas de saúde. Prom Saúde 2002; 3: 31-6.

3. Serruya S, Lago TG. A mortalidade materna no Brasil. J Febrasgo 2001; 8: 6-8
4. BEMFAM (Sociedade Civil Bem Estar no Brasil). Pesquisa Nacional sobre Demografia e Saúde: 1996. Rio de Janeiro: A Sociedade; 1997.

5. OPAS (Organização Pan-Americana da Saúde). Rede Interagencial de Informações para a Saúde. Indicadores básicos para a saúde no Brasil: conceitos e aplicações. Brasília (DF): A Organização; 2002.

6. Ministério da Saúde. Centro Nacional de Epidemiologia. Séries históricas. Disponível no site www.funasa.gov.br 
[dia 16 Fev. 2003]

7. Vianna SM, Nunes A, Santos JR, Barata RB. Medindo as desigualdades em saúde no Brasil: uma proposta de monitoramento. Brasília (DF): OPAS; 2001.

8. Donabedian A. The seven pillars of quality. Arch Pathol Lab Med 1990; 114: 1115-8.

9. Davis-Floyd R. The technocratic, humanistic, and holistic paradigms of childbirth. Int J Gynecol Obstet 2001; 48 Suppl: S33-S52.

10. Murphy-Lawlees J. A history of obstetric thinking. Bloomington: Indiana University College; 1998.

11. Illich I. A expropriação da saúde. Nêmesis da medicina. Rio de Janeiro: Nova Fronteira; 1975. p.33.

12. Foucault M. Microfísica do poder. 12. ed. Rio de Janeiro: Graal; 1996.

13. Serruya S. Mulheres esterilizadas: submissão e desejo. Belém: Universidade Estadual do Pará; 1996.

14. Trostle J. Algunas reflexiones acerca de las ciencias sociales y biomédicas: ¿dos catedrales o un arquétipo inconcebible? Experiencias inovadoras en salud reproductiva. La complementación de las ciencias médicas y sociales. Buenos Aires: CEDES; 2002.

15. World Health Organization. Care in normal birth: a practical guide. Geneva: The Organization; 1996.

16. Ávila MBM, Corrêa S. O movimento de saúde e direitos reprodutivos no Brasil: revisitando percursos. In: Galvão L, Diaz J., organizadores. Saúde sexual e reprodutiva no Brasil. São Paulo: Hucitec; 1999. p.70-103.

17. Diniz CSG. Maternidade avoluntária, prazerosa e socialmente amparada: breve história de uma luta. In: Coletivo Feminista Sexualidade e Saúde. Saúde das mulheres: experiência e prática. São Paulo: O Coletivo; 2000. p. 22

18. Diniz CSG, D'Oliveira AFPL. Gender violence and reproductive health. Int J Gynecol Obstet 1998; 63 [Supp11]: S33-S42.

19. Ministério da Saúde. Secretaria de Políticas de Saúde. Parto, aborto e puerpério: assistência humanizada à mulher. Brasília (DF): O Ministério; 2001.

20. Wagner M. Fish can't see water: the need to humanize birth. Int J Gynecol Obstet 2001; 48 [Suppl ]: S33-S52.

21. Mulrow CD. The medical review article: state of the science. Ann Intern Med 1987; 106: 185-8.
22. Umenai T, Wagner M, Page LA, Faundes A, Rattner D, Dias MA, Tyrrell MA, Hotimsky S, Haneda K, Onoki D, Mori T, Sadamori T, Fujiwara M, Kikuchi S. Conference agreement on the definition of humanization and humanized care. Int J Gynecol Obstet 2001; 75: S3-S4.

23. Ministério da Saúde. Secretaria de Políticas de Saúde. Programa de humanização no pré-natal e nascimento. Brasília (DF): O Ministério; 2000.

24. Serruya S, Cecatti JG, Lago TG. O Programa de Humanização no Pré-Natal e Nascimento (PHPN) do Ministério da Saúde no Brasil: resultados iniciais. Cad Saúde Pública 2004: 20 (5) [in press].

25. Gharoro EP, Okonkwo CA. Changes in service organization: antenatal care policy to improve attendance and reduce maternal mortality. Int J Gynecol Obstet 1999; 67: 179-81.

26. Pitanguy JO. Movimento Nacional e Internacional de Saúde e Direitos Reprodutivos. In: Giffin K, Costa SH. Questões da saúde reprodutiva. Rio de Janeiro: FIOCRUZ; 1999.

27. Abouzahr C, Wardlaw T. La mortalidad materna al término de una década: hay indicios de progreso? Bul World Health Org 2001; 79: 561-8.

28. Campbell O. What are maternal health policies in developing countries and who drives them? A review of the last half century. In: Brouwere VD, Lerberghe WV. Safe motherhood strategies: a review of the evidence. Brussels: ITG Press; 2001. p. 415-48. (Studies in Health Services Organization and Policy).

29. Lerberghe WV, Brouwere V. Reducing maternal mortality in a context of poverty. In: Brouwere VD, Lerberghe WV. Safe motherhood strategies: a review of the evidence. Brussels: ITG Press; 2001. p.1-5. (Studies in Health Services Organization and Policy).

30. Ribeiro JM, Costa NR, Pinto LFS, Silva PLB. Atenção ao pré-natal na percepção das usuárias do Sistema Único de Saúde: um estudo comparativo. Cad Saúde Publica 2004; 20: 534-45.

31. Ministério da Saúde. Indicadores da assistência à saúde. Disponível em: www.saude.gov.br [2003 12 fev 2003].

Recebido em 31 de dezembro de 2004

Versão final apresentada em 24 de maio de 2004

Aprovado em 22 de julho de 2004 